\title{
Performance Evaluation of HE, AHE and Fuzzy Image Enhancement
}

\author{
Sargun \\ Student M.Tech (ECE) \\ Guru Nanak Dev University \\ Regional Campus Gurdaspur
}

\author{
Shashi B. Rana \\ Asstt Professor \\ Guru Nanak Dev University \\ Regional Campus Gurdaspur
}

\begin{abstract}
Image enhancement plays an important role in vision applications. Enhancement is the technique of improving the superiority of a digital stored image. Recently much work is performed in the field of images enhancement. Many techniques have already been proposed up to now for enhancing the digital images. In this paper, we have compared three basic techniques of the image enhancement which are histogram equalization, adaptive histogram equalization and fuzzy enhancement. From the comparison, it has been proved that the fuzzy enhancement performs much better as compared to histogram and adaptive histogram equalization.
\end{abstract}

\section{Keywords}

Image Enhancement, Histogram Equalization, Adaptive Histogram Equalization, Fuzzy Enhancement.

\section{INTRODUCTION}

Image Enhancement is essentially a simplest and attractive area of digital image processing. Image enhancement is method used to enhance the overall superiority of the corrupted images can be attained by using enhancement mechanisms. So that the human eye can naturally detect the key features of the pictures. It is used to eliminate the inappropriate artifacts from the pictures like noise or brighten the photograph and it simply to identify main features and then it looks improved. It is an individual area of digital image processing. To create a graphic display further helpful to visualize and examination, it recover the photograph features such as edges or boundaries. It enlarges the dynamic range of collected features. It does not increase the inbuilt content of data.

\section{IMAGE ENHANCEMENT TECHNIQUES}

\subsection{Histogram Equalization}

Histogram equalization is the method of image enhancement that is used to enhance the contrast of images. In histogram equalization it is not compulsory that the contrast of an image will always be raised. Sometimes it shows that it can be not as good as than the contrast of an image reduced. Before working with histogram equalization it's necessary to recognize the two main concepts of histogram equalization that are known as PMF (probability mass function) and CDF (cumulative distributive function). First of all estimate the $\mathrm{PMF}$ and CDF for all pixels in an image then work further. The transformation $\mathrm{T}(\mathrm{r})$ needed to be obtaining by using:

$$
s=T(r)=p_{r}(r) d w=c d f_{i}
$$

Histogram equalization is further divided into two broad categories: a) Local Histogram Equalization: The overall contrast of an image can be improved efficiently.

b) Global Histogram Equalization: Based on grey level content of an image, the pixels are modified by transformation function.

Histogram equalization is a point process. In order to obtain a uniform histogram for an image the point process redistributes the image's intensity distribution.

Histogram equalization can be done in three main steps:

1. Compute the histogram of an image.

2. Calculate the normalized sum of histogram.

3. Transform the input image to an output image.

Various advantages of Histogram Equalization are that it is finest for visual perception mostly when image has close contrast data. Moreover the purpose of this method developed best outcomes for radiographic and thermal images. The main limitation of this method is the noise amplification when the image has major low intensity area.

\subsection{Adaptive Histogram Equalization}

Adaptive histogram equalization $[\mathrm{AHE}]$ is a computer image processing technique used to recover contrast of the pictures. It is a sparkling contrast enhancement for both natural images and medical images and other initially non visual images. It differs from ordinary histogram equalization $[\mathrm{HE}]$ in the respect that the adaptive method computes several histograms, each corresponding to a distinct section of the image, and uses them to redistribute lightness value of the image. In image fusion process, fusion process may degrade the sharpness of the fused image so to overcome this problem of poor brightness adaptive histogram equalization will be used to enhance the results further. We can say that adaptive histogram equalization will come in action to preserve the brightness of the fused image.. The main point of AHE is that in which at smaller scales contrast of an image is enhanced; while at larger scales contrast of an image is reduced or decreased. The advantage of adaptive histogram equalization [AHE] is that it is automatic, reducible, and locally adaptive and usually produces superior images. This is the most excellent method to apply when global histogram equalization cannot produce best outcomes.

\section{LITERATURE SURVEY}

P.jaatheeswari et al. [1] has presented that by histogram equalization, contrast enhancement of an image can be effectively worked. On the other hand, this technique to produce irrelevant visual deterioration likes saturation effect. To overcome this drawback is by preserving the mean brightness of input image inside the output image. In this 
paper for image contrast enhancement and brightness preservation, introduce a new technique contrast stretching recursively separated histogram equalization. Two stages of algorithm are to be applied. Different keywords that were used in this paper like image contrast enhancement, contrast stretching, image contrast enhancement and histogram equalization. Sun et al. [2] has shown that to automatically produce bas-reliefs based on adaptive histogram equalization, starting from an input height field. A network model may on the other hand be provided, in which case a height field was first created via orthogonal or perspective projection. The height field has been regularly gridded and treated as an image, enabling a modified adaptive histogram equalization technique to be used to produce a bas-relief with a userchosen height range. Alter the input image-contrastenhancement adaptive histogram equalization technique to use gradient weights also to enhance the shape features of the basrelief. To efficiently compact the height field, limit the heightdependent scaling factors used to calculate relative height variations in the outcome from height variations in the input; this protects any height differences from having too huge result. Outcomes of adaptive histogram equalization over different neighborhood sizes are averaged to protect data at different scales in the resulting bas-relief. Compared to previous approaches, the proposed algorithm is simple and yet largely preserves original shape features. Experiments show that our results are, in general, comparable to and in some cases better than the best previously published methods. Demirel et al. [3] has provided a novel satellite image contrast enhancement method based on the discrete wavelet transform and singular value decomposition has been projected. In this method by using discrete wavelet transform divide the input image into the four frequencies subbands and estimates the singular value matrix of low-low subband image and then restructure improved by applying inverse discrete wavelet transform. The illustration results on the finishing image quality show the advantage of the projected technique over the predictable and the state-of-the-art method. The different techniques used for example discrete wavelet transform, Image equalization and satellite image contrast enhancement. Compare the techniques with general histogram equalization and local histogram equalization. Murahira et al. [4] has proved for improving images histogram equalization is one of the general technique. On the other hand, it will cause a consequence on the brightness saturation or shadow in several identical areas. To overcome these things mean preserving bi-histogram equalization technique has been developed. New histogram equalization with variable enhancement degree and bi-histogram equalization with variable degree has developed. By only one parameter the degree of every of these techniques has controlled. Every type of images is enhanced effectively. The outcomes show that especially, bi-histogram equalization with variable degree can recognize the normal enhancement. Iyatomi et al. [5] has proved a perfect color information in dermoscopy images has very imperative for melanoma diagnosis because improper white balance or brightness in the images unfavorably affects the diagnostic performance. It has been offered an automated color normalization technique for dermoscopy images of skin lesions. In this paper based on a total of 319 images color normalization filters has been developed, which stabilize color of images using the Hue saturation intensity color method. We determined that the color uniqueness of the secondary part of the tumors appreciably manipulates the color normalization and established that the developed normalization filter achieved acceptable normalization presentation the same as evaluated by a cross-validation test. Roomi and Prabhu et al.
[6] provided that for better visualization of low contrast images contrast enhancement method has been used. Histogram equalization used for Contrast enhancement. Histogram equalization has not suitable for consumer electronics product straightforwardly. It provides a new method of histogram equalization that tries to found foreground and background pixels of an image and apply bihistogram equalization on them. Its outcomes shows that this algorithm preserves the original image as compare to other techniques. Josephus and Remya S [7] proved that for local content emphasis that the adaptive histogram equalization has the best and efficient algorithm. But sometimes has a problem of amplification and introduction of the speckle noise due to it information lost. To overcome this problem the multilayered contrast limited adaptive histogram equalization with frost filter that focused on application to medical images. In this on contrast limited adaptive histogram equalization the combination of frost and median filter both has been used. For the removal of speckle noise in images the technique of frost filter has been done. The work has been done on medical images such as mammogram, knee, and brain images. Jeong CB et al. [8] presented the most effective method of image enhancement based on histograms, which may assist radiologists in interpreting successive whole-body bone scans effectively. Forty-eight successive whole-body bone scans from 10 patients were obtained and evaluated using six methods of image enhancement based on histograms: histogram equalization, brightness-preserving bi-histogram equalization, contrast-limited adaptive histogram equalization, end-in search, histogram matching, and exact histogram matching. Comparison of the results of the different methods was made using three similarity measures peak signal-to-noise ratio, histogram intersection, and structural similarity. Image enhancement of successive bone scans using exact histogram matching showed the best results out of the six methods measured for all similarity measures. Exact histogram matching is the best method of image enhancement based on histograms for diagnosing successive whole-body bone scans. The method for successive whole-body bone scans has the potential to greatly assist radiologists quantify interval changes more accurately and quickly by compensating for the variable nature of intensity information. Consequently, it can improve radiologists' diagnostic accuracy as well as reduce reading time for detecting interval changes. Sundaram, M. [9] presented Modified Contrast Limited Adaptive Histogram Equalization. Histogram equalization is an effective and simple technique for contrast enhancement. The conventional histogram equalization (HE) usually results in excessive contrast enhancement because of insufficient control on the degree of enhancement. The Histogram Modified Contrast Limited Adaptive Histogram Equalization adjusts the amount of contrast enhancement, which in turn provides the resultant image a strong contrast and brings the neighborhood details for more relevant interpretation. It incorporates both histogram modifications being an optimization technique and Contrast Limited Adaptive Histogram Equalization. This method is tested for Mias mammogram images. The performance of this approach has been determined utilizing the parameter like Enhancement Measure (EME). From the subjective and quantitative measures it is interesting this proposed technique provided better contrast enhancement with preserving the neighborhood information of the mammogram images. Ahmed et al. [10] has explained that our learning uncovers that HE - in a remarkable contrast to its claim, is not associated to enhancement of contrast. To recognize this observation, we begin through real world images which have 
variable amount of image quality that almost consistently want processing to get better image contrast. For this reason, $\mathrm{HE}$ is used upon technique. HE is working with grey level of images. As a result, the learning aims to get out the realistic nature of alteration functions used by histogram equalization. To recognize these calculations, this paper dismantles histogram equalization into its building blocks. These blocks show the relationship between fundamentals and contrast of Histogram Equalization. In this different keywords are used like Histogram equalization, Cumulative density function, probability density, contrast. Amina saleem et al. [11] has planned a scheme that balances the situation of local and global contrast enhancements and a reasonable illustration of the original image and defeat the limitations of altered contrast enhancement that is fusion-based contrast enhancement algorithms. By using laplacian pyramid decomposition techniques has used for fusion. The results show that enhancing the local and global contrasts. Jha et al. [12] in this paper a contrast enhancement method by means of scaling of internal noise of a dark image in discrete cosine transform domain has been projected. The method of enhancement recognized to noise-induced transition of discrete wavelet transform coefficients from a condensed state to an improved state. This conversion has been effected by the inner noise there due to shortage of enough explanation and can be modeled by a general bistable method exhibiting dynamic stochastic resonance. The projected method adopts a local adaptive processing and extensively enhances the image disparity and color information although ascertaining superior perceptual feature. After compared with the presented enhancement methods like adaptive histogram equalization, gamma correction, single-scale retinex, multiscale retinex, modified high-pass filtering, multi-contrast enhancement, multi-contrast enhancement with dynamic range compression, color enhancement by scaling, edge-preserving multi-scale decomposition and automatic controls of popular imaging tool, the projected method gives outstanding presentation in conditions of relative contrast enhancement, colorfulness and visual superiority of enhanced image. Different techniques have been used in this paper such as Contrast enhancement, dynamic stochastic resonance, bistable system, double well parameters. $\mathrm{Xu}$, Hongteng et al. [13] proposed a story histogram-based model for contrast enhancement ;.Based on their analysis in regards to the relationships of histogram with contrast, they establish a style which achieved contrast enhancement by an optimum transform of histogram, gave two metrics called contrast gain and nonlinearity of transform to gauge the strength of enhancement and the seriousness of distortion caused by enhancement respectively. The ratio of the 2 proposed metrics not merely gives guidance for the configuration of parameter in the algorithm, but in addition provides a helpful measurement for contrast distortion, which can be a potential means to fix judge whether the contrast of an image is optimal. Experimental results showed the superior performances of the proposed algorithm in image enhancement. Khairunnisa Hasikin et al. [14] In this paper a fluffy grayscale improvement strategy for low differentiation picture has been proposed. The corruption of the low difference picture is essentially created by the insufficient lighting amid picture catching and in this manner at last brings about non -uniform brightening in the picture. The vast majority of the created difference improvement procedures enhanced picture quality without considering the non-uniform lighting in the picture. The fluffy grayscale picture improvement method is proposed by amplifying fluffy measures contained in the picture. The participation capacity was then altered to improve the picture by utilizing force law change and immersion administrator.. Weitao Zheng, et al. [15] In this paper a contourlet change and PCNN based picture improvement calculation has been proposed. This strategy defeats the customary wavelet changes inadequacies in scanty speaking to interwoven semantic points of interest of pictures, for example, multidirectional edges. Firstly the pictures were decayed into multi-directional and multi-scale shape portions by the contourlet change, the headings of the line-formed intermittence in the pictures were discovered. Also, another physiological improvement capacity focused around the beat coupled neural systems ( $\mathrm{PCNN}$ ) was proposed to improve the coefficients by contourlet change. Finally, the upgraded coefficients were recreated and the improved aftereffects of the first pictures were gotten, the system was contrasted and ordinary differentiation upgrade techniques, for example, the histogram adjustment. The results have demonstrated that proposed system was superior to other in improving the directional line-molded items. Yingjie Zhang[16] In this paper a novel calculation for picture upgrade and denoising focused around anisotropic dispersion and fluffy rationale hypothesis is proposed. The greatest fluffy entropy standard has been utilized to guide the standardized picture to the fluffy area. At that point, the picture was separated by the partial anisotropic dissemination process. The exploratory results demonstrated that the proposed strategy have evacuated commotion and improve differentiate sufficiently and fine points of interest of bore extension pictures were generally saved.

\section{RESULTS AND DISCUSSIONS}

\subsection{Experimental Setup}

In order to compare the histogram equalization, adaptive histogram equalization and fuzzy enhanced algorithm, design and implementation has been done in MATLAB using image processing toolbox.

Figure 1 represents the input image.

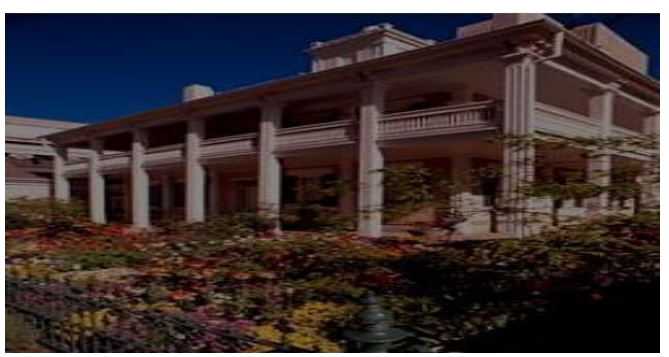

Fig 1: Input Image

Figure 2 represents the results of the histogram equalization on the input image.

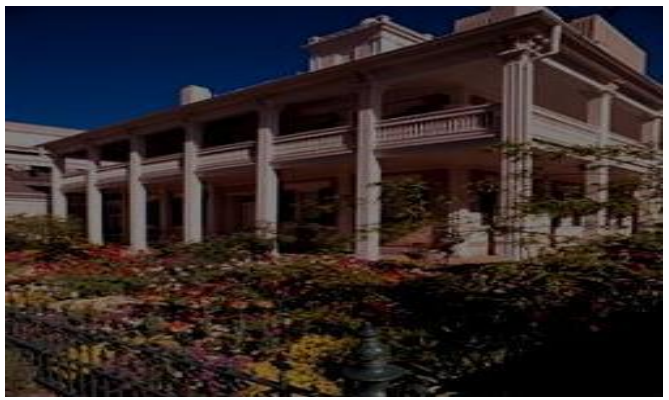

Fig 2: Results of the histogram equalization 
Figure 3 represents the results of the adaptive histogram equalization on the input image.

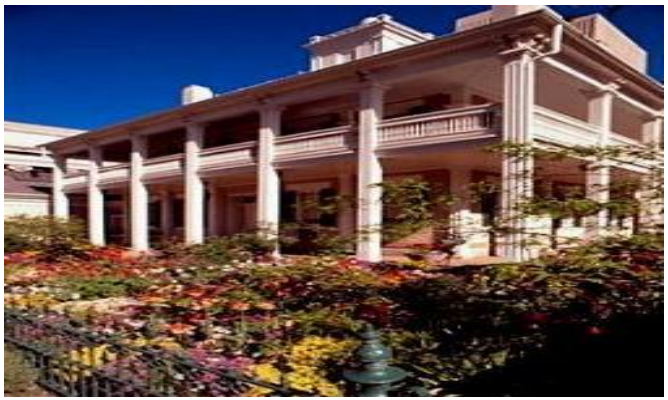

Fig 3: Results of the adaptive histogram equalization

Figure 4 represents the results of the fuzzy enhancement on the input image.

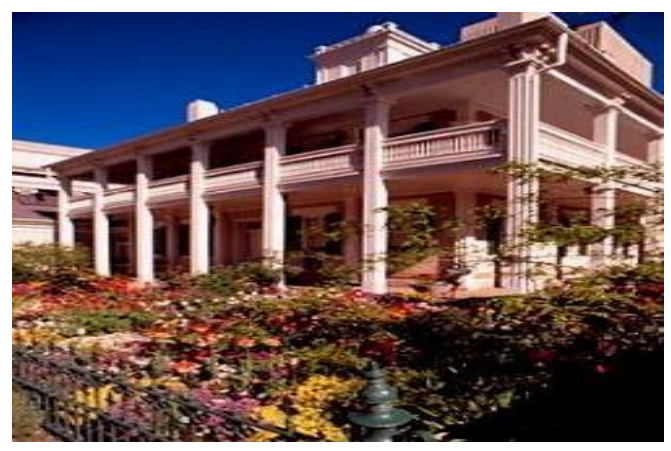

Fig 4: Results of the fuzzy enhancement

\section{PERFORMANCE EVLAUATION}

\subsection{Mean Square Error (MSE)}

In image processing mean square error is the most general measure for performance measurement of the existing method and the coded images. It is computed by using equation

$$
M S E=\frac{1}{M N} \sum_{i=1}^{M} \sum_{j=1}^{N}\left(f(i, j)-f^{\prime}(i, j)\right)^{2}
$$

Table 1 is showing the quantized analysis of the mean square error. As mean square error needs to be reduced therefore the fuzzy enhancement algorithm is showing the better results than the available methods as mean square error is less in every case. The mean Square error is reduced in each case. The method is tested on the number of images and in each case shows the better results than the histogram and the adaptive histogram method.

Table 1: MSE Evaluation

\begin{tabular}{|c|c|c|c|}
\hline IMAGES & $\begin{array}{c}\text { Histogram } \\
\text { Equalization }\end{array}$ & $\begin{array}{c}\text { Adaptive } \\
\text { histogram } \\
\text { equalization }\end{array}$ & $\begin{array}{c}\text { Fuzzy Image } \\
\text { Enhancement }\end{array}$ \\
\hline 1 & 2344.200000 & 5563.700000 & 215.6498 \\
\hline 2 & 2997.800000 & 3768.800000 & 275.4817 \\
\hline 3 & 3239.800000 & 9117.400000 & 297.5358 \\
\hline 4 & 1056.100000 & 1251.700000 & 98.1910 \\
\hline 5 & 2643.100000 & 3045.100000 & 242.4858 \\
\hline 6 & 2322.000000 & 4629.700000 & 213.7253 \\
\hline 7 & 2233.700000 & 1512.300000 & 206.6683 \\
\hline 8 & 1534.900000 & 5902.300000 & 141.7540 \\
\hline 9 & 1051.500000 & 1435.200000 & 955.1944 \\
\hline 10 & 1027.500000 & 2129.500000 & 935.2309 \\
\hline
\end{tabular}

Figure 5 has shown the quantized analysis of the mean square error of different images. It is very clear from the graph that there is decrease in MSE value of images with the use of method over existing methodS. This decrease represents improvement in the objective quality of the image.

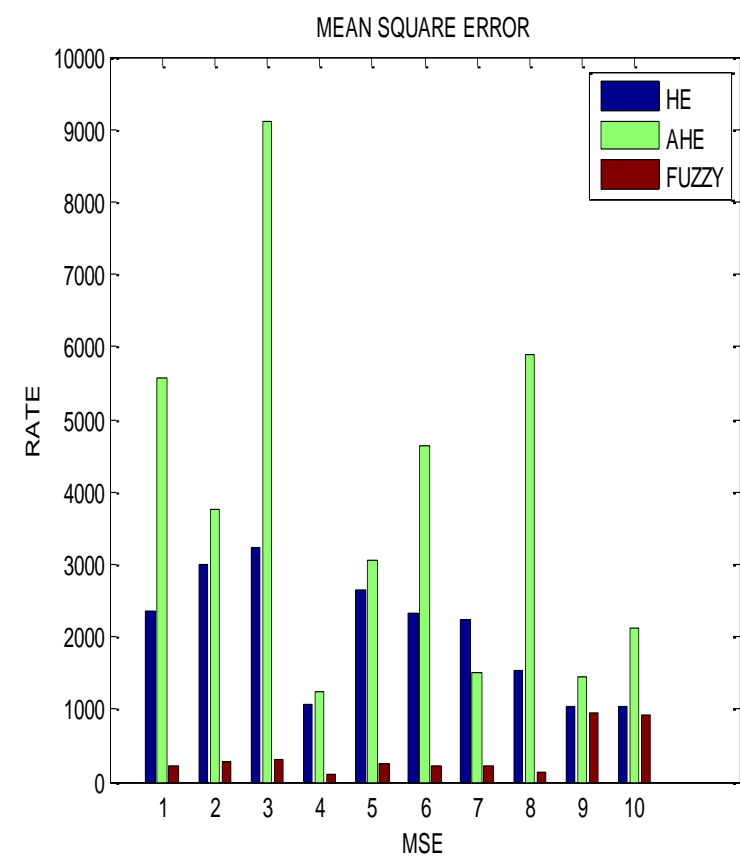

Figure 5: MSE Evaluation

\subsection{Peak Signal to Noise Ratio (PSNR)}

Peak signal to noise ratio measure the degree of image distortion. PSNR is used to measure the quality between the original image and compressed image.

PSNR is defined as:

$$
P S N R=10 . \log _{10}\left(\frac{M A X_{I}^{2}}{M S E}\right)
$$

Table 2 is showing the comparative analysis of the Peak Signal to Noise Ratio (PSNR). As PSNR need to be maximized; so the main goal is to increase the PSNR as much as possible. Table 2 has clearly shown that the PSNR is maximum in the case of the proposed technique; therefore algorithm is providing better results than the available methods. The method is tested on the number of images and in each case shows the better results than the existing method.

Table 2: PSNR Evaluation

\begin{tabular}{|c|c|c|c|}
\hline IMAGES & $\begin{array}{c}\text { Histogram } \\
\text { Equalization }\end{array}$ & $\begin{array}{c}\text { Adaptive } \\
\text { histogram } \\
\text { equalization }\end{array}$ & $\begin{array}{c}\text { Fuzzy Image } \\
\text { Enhancement }\end{array}$ \\
\hline 1 & 14.4308 & 10.6771 & 24.7933 \\
\hline 2 & 13.3627 & 12.3688 & 23.7299 \\
\hline 3 & 13.0256 & 8.5321 & 23.3954 \\
\hline 4 & 17.8936 & 7.1558 & 28.2101 \\
\hline 5 & 13.9096 & 13.2948 & 24.2839 \\
\hline 6 & 14.4722 & 11.4753 & 24.8322 \\
\hline 7 & 14.6406 & 6.3345 & 24.9781 \\
\hline 8 & 16.2701 & 10.4206 & 26.6154 \\
\hline 9 & 7.9129 & 16.5618 & 18.3299 \\
\hline 10 & 8.0129 & 14.8480 & 18.4216 \\
\hline
\end{tabular}


Figure 6 has shown the quantized analysis of the peak signal to noise ratio of different. It is very clear from the plot that there is increase in PSNR value of images with the use of method over existing methods. This increase represents improvement in the objective quality of the image.

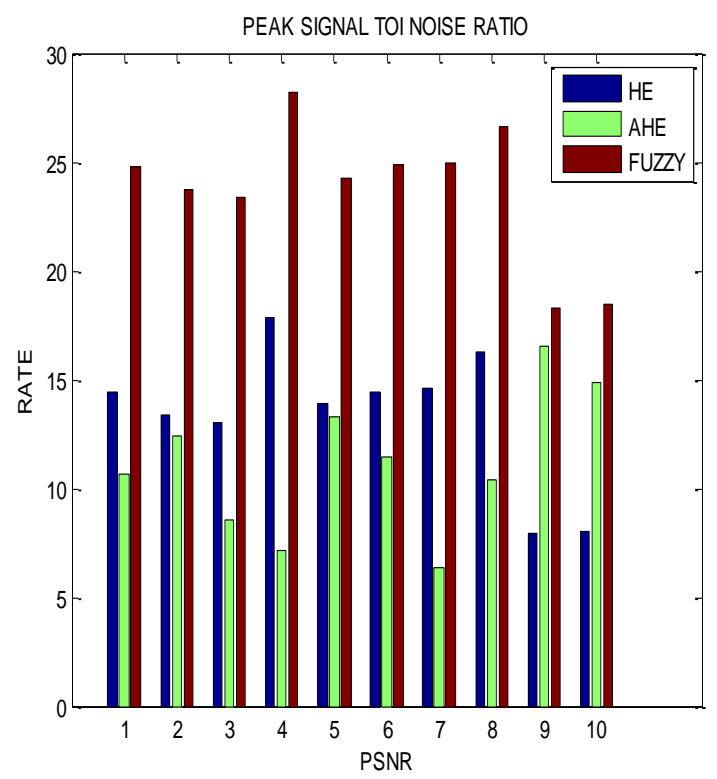

Figure 6: PSNR Evaluation

\subsection{Root Mean Squared Error (RMSE):}

RMSE is the measure of differences between values predicted by a model or an estimator and the values actually observed. Basically, the RMSE represents the sample standard deviation of the differences between input image and the final fog free image. Root mean square error is calculated by the given formulae

$$
R M S E=\frac{\Sigma\left(f\left(x_{i}\right)-y_{i}\right)^{2}}{n}
$$

Table 3 is showing the comparative analysis of the root mean square error. Table has clearly shown that is less in fuzzy enhancement results therefore the algorithm has shown significant results over the histogram and adaptive histogram algorithm.

Table 3: RMSE Evaluation

\begin{tabular}{|c|c|c|c|}
\hline IMAGES & $\begin{array}{c}\text { Histogram } \\
\text { Equalization }\end{array}$ & $\begin{array}{c}\text { Adaptive } \\
\text { histogram } \\
\text { equalization }\end{array}$ & $\begin{array}{c}\text { Fuzzy Image } \\
\text { Enhancement }\end{array}$ \\
\hline 1 & 48.4170 & 74.5904 & 14.6850 \\
\hline 2 & 54.7526 & 61.3902 & 16.5976 \\
\hline 3 & 56.9191 & 95.4851 & 17.2492 \\
\hline 4 & 32.4981 & 111.8797 & 9.9091 \\
\hline 5 & 51.4115 & 55.1821 & 15.5720 \\
\hline 6 & 48.1871 & 68.0415 & 14.6193 \\
\hline 7 & 47.2620 & 122.9741 & 14.3760 \\
\hline 8 & 39.1775 & 76.8262 & 11.9061 \\
\hline 9 & 102.5409 & 37.8836 & 30.9062 \\
\hline 10 & 101.3667 & 46.1465 & 30.5815 \\
\hline
\end{tabular}

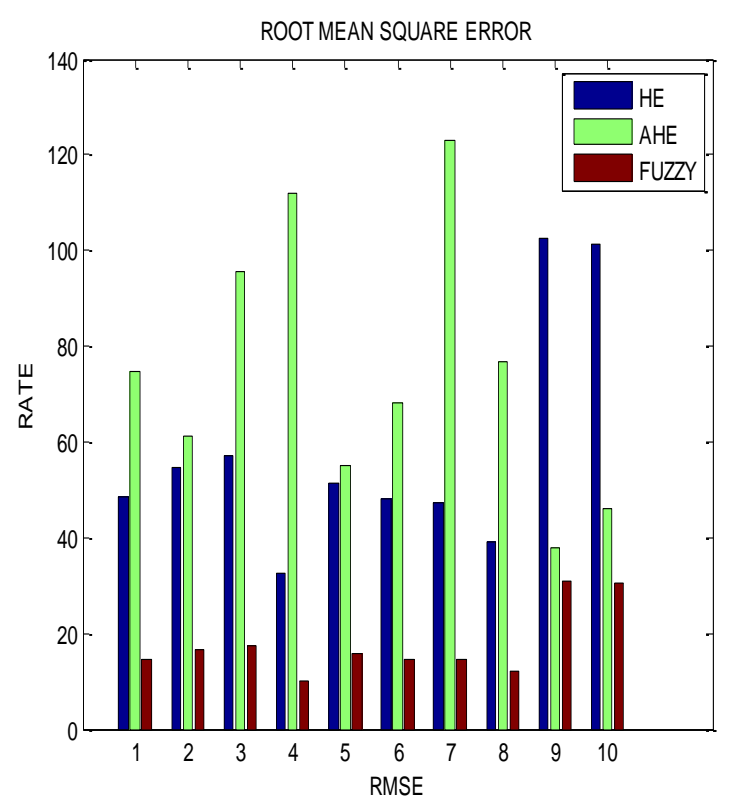

Figure 7: RMSE Evaluation

Figure 7 has shown the quantized analysis of the Root mean squared Error of different images. It is very clear from the plot that there is decrease in RMSE value of images with the use of method over existing method. This decrease represents improvement in the objective quality of the image

\section{CONCLUSION}

In this paper, we have compared three basic techniques of the image enhancement which are histogram equalization, adaptive histogram equalization and fuzzy enhancement. The comparison has been made on the basis of various performance metrics like mean square error, peak signal to noise ratio and root mean square ration. From the comparison, it has been proved that the fuzzy enhancement performs much better as compared to histogram and adaptive histogram equalization.

\section{REFERENCES}

[1] Jagatheeswari, P., S. Suresh Kumar, and M. Rajaram. "Contrast Stretching Recursively Separated Histogram Equalization for Brightness Preservation and Contrast Enhancement." Advances in Computing, Control, \& Telecommunication Technologies, 2009. ACT'09. International Conference on. IEEE, 2009.

[2] Sun, Xianfang, et al. "Bas-relief generation using adaptive histogram equalization." Visualization and Computer Graphics, IEEE, 2009.

[3] Demire1, Hasan, Cagri Ozcinar, and Gholamreza Anbarjafari. "Sate1lite image contrast enhancement using discrete wavelet transform and singular value decomposition." Geoscience and Remote Sensing 1etters, IEEE 7.2: pp.333-337, 2010.

[4] Murahira, Kota, Takashi Kawakami, and Akira Taguchi. "Modified histogram equalization for image contrast enhancement." Communications, Control and Signal Processing (ISCCSP), 2010 4th International Symposium on. IEEE, 2010. 
[5] Iyatomi, Hitoshi, et al. "Automated color normalization for dermoscopy images." Image Processing (ICIP), 2010 17th IEEE International Conference on. IEEE, 2010.

[6] Maragatham, G., S. Md Mansoor Roomi, and T. Manoj Prabu. "Contrast enhancement by object based Histogram Equalization." Information and Communication Technologies (WICT), 2011 Wor1d Congress on. IEEE, 2011.

[7] Josephus, Chelsy Sapna, and S. Remya. "Multilayered Contrast limited Adaptive Histogram Equalization Using Frost Filter." Recent Advances in Intelligent Computational Systems (RAICS), 2011 IEEE. IEEE, 2011.

[8] Jeong, Chang $\mathrm{Bu}$, et a1. "Comparison of image enhancement methods for the effective diagnosis in successive whole-body bone scans." Journa1 of digita1 imaging 24.3,2011.

[9] Sundaram, M., K. Ramar, N. Arumugam, and G. Prabin. "Histogram based contrast enhancement for mammogram images." In Signal Processing, Communication, Computing and Networking Technologies (ICSCCN), 2011 International Conference on, pp. 842-846. IEEE, 2011.

[10] Ahmed, M. Mahmood, and Jasni Mohamad Zain. "A Study on the Validation of Histogram Equalization as a Contrast Enhancement Technique." Advanced Computer Science Applications and Technologies (ACSAT), 2012 Internationa1 Conference on. IEEE, 2012.
[11] Soliman, Omar S., and A. S. Mahmoud. "A classification system for remote sensing satellite images using support vector machine with non-linear kernel functions." Informatics and Systems (INFOS), 2012 8th International Conferences on. IEEE, 2012.

[12] Jha, Rajib Kumar, et al. "Internal noise-induced contrast enhancement of dark images." Image Processing (ICIP), 2012 19th IEEE International Conference on. IEEE, 2012.

[13] Xu, Hongteng, Guangtao Zhai, and Xiaokang Yang. "No reference measurement of contrast distortion and optimal contrast enhancement." InPattern Recognition (ICPR), 2012 21st International Conference on, pp. 1981-1984. IEEE, 2012.

[14] Khairunnisa Hasikin, Nor Ashidi Mat Isa "Enhancement of the low contrast image us-ing fuzzy set theory ", In IEEE 14th International Conference on Modelling and Simulation , pp.371-376, 2012.

[15] Weitao Zheng, Tian Pu, Jian Cheng, Hu Zheng "Image contrast enhancement by con-tourlet transform and PCNN ", In IEEE International Conference on Audio, Language and Image Processing (ICALIP) , pp.735-739, 2012.

[16] Yingjie Zhang "A Novel Contrast Enhancement and Denoising Method for Borescope Images ", In IEEE fifth International Conference on Advanced Computational Intelligence(ICACI) pp.570-573, October 2012. 